\title{
Modelo experimental para treinamento com dispositivo de assistência ventricular pulsátil
}

\author{
Maurício GALANTIER *, Geisha MOREIRA *, José Luiz GHIOTTO *, João GALANTIER *,
} Selma Aparecida A. IANONI *, Lorinaldo Lopes de MORAES *, Jozef FÉHER *

RBCCV 44205-269

GALANTIER, M.; MOREIRA, G.; GHIOTTO, J.L.; GALANTIER, J.; IANONI, S. A. A.; MORAES, L. L.; FËHER, J.- Modelo experimental para treinamento com dispositivo de assistência ventricular. Rev. Bras. Cir. Cardiovasc., $10(3): 133-138,1995$.

RESUMO: Os dispositivos de Assistência Ventricular (D A V) têm sido utilizados para permitir a manutençăo de condiçōes cardiocirculatórias em dois tipos de situações: 1-) Como ponte para transplante cardiaco em paciente candidato e que, na espera de doador apropriado, tem suas condiçōes cardiocirculatórias muito deterioradas. 2-) Como suporte circulatório em situaçōes agudas em que se prevê uma possivel recuperaçăo funcional do coraçåo, como em miocardites agudas, miocardiopatia puerperal, falência miocárdica pós cardiotomia, rejeiçāo aguda pós transplante cardiaco etc. Entre os vários dispositivos utilizados, o modelo descrito por Pierce-Donachy, desenvolvido pela Thoratec Laboratories Corporation, consiste em aparelho de fluxo pulsátil, pneumático, externo, podendo ser usado como suporte uni ou biventricular. O ventriculo artificial é acionado por um módulo que permite vários ajustes manuais ou mesmo automáticos para melhor adequaçāo as diferentes condiçóes clínicas do paciente. O treinamento para utilizaçăo do dispositivo implica na integraçāo de vários setores, como cirurgiōes, intensivistas, enfermeiras, biomédicos e eletrotécnicos, necessitando laboratório especializado, como uso de animais de maior porte, de custo elevado e manuseio complicado, dificultando a repetiçāo freqüente dos experimentos. Os autores desenvolveram um modelo experimental, constituido por um coraçăo bovino conectado pelo átrio esquerdo e pela aorta a um circuito de circulação extracorpórea. Através de técnica cirúrgica padronizada, sāo instaladas as cânulas apropriadas: as mesmas são conectadas ao ventriculo artificial e este ao módulo de acionamento. Com este modelo podese, de maneira simples, didática e reprodutivel, efetuar o treinamento de aspectos técnicos de implante, manuseio de módulo de acionamento e simulaçäo de situaçóes clínicas e emergenciais.

DESCRITORES: Assistência ventricular, experimental. Circulaçăo assistida, mecånica, experimental.

\section{INTRODUÇÃO}

Os Dispositivos de Assistência Ventricular (D A V) vêm sendo utilizados desde $1963^{10}$, para permitir a manutençăo das condiçōes cardiocirculatórias em pacientes candidatos a transplante cardia$\mathrm{co}$, em fase crítica de sua evolução, e que não têm, de imediato, um doador disponivel (ponte para transplante cardiaco) $1,3,8,15$, ou em pacientes com quadro agudo de falência miocárdica (miocardiopatia aguda, infarto agudo do miocárdio, falência na recuperação pós correção cirúrgica adequada, rejeição pós transplante $2,9,14,20$, permitindo um período de readaptação após o qual as condições miocárdicas venham a se recuperar, possibilitando a retirada do sistema (Quadro 1).

Existem, basicamente, dois sistemas mecâni-

Trabaiho realizado na Escola de Enfermagem do Hospital Israelita Albert Einstein. São Paulo, SP, Brasil. Apresentado ao $22^{\circ}$ Congresso Nacional de Cirurgia Cardiaca. Brasilia, DF, 30 de março a $1^{2}$ de abril, 1995.

"Do Hospital Israelita Albert Elnstein.

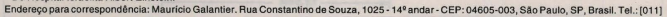
$543.1640 / 842.3265$ 


\section{QUADRO 1}

\section{DISPOSITIVOS DE ASSISTÊNCIA VENTRICULAR \\ 1 - Ponte para transplante cardiaco \\ 2 - Recuperação Miocárdica \\ Miocardiopatia Aguda \\ Infarto Agudo do Miocárdio \\ Falência Miocárdica Pós Cardiotomia \\ Rejeiçăo Aguda Pós Transplante}

cos para assistência circulatória: os de fluxo continuo e os de fluxo pulsátil ${ }^{13}$, sendo que, para estes últimos, o sistema de acionamento pode ser pneumático ou eletromagnético. Mais recentemente, temse procurado desenvolver sistemas totalmente implantáveis 7 , permitindo ao paciente em ponte para transplante maior autonomia, inclusive possibilitando sua vida fora do ambiente hospitalar (Quadro 2).

\section{QUADRO 2}

\section{DISPOSITIVOS DE ASSISTÊNCIA VENTRICULAR}

Fluxo Continuo

Bombas do Roletes

Bombas Centrifugas

Fluxo Pulsátil

Pneumáticos

Eletromagnéticos

Externos

Parcialmente Implantáveis

Totalmente Implantáveis

Um dos sistemas utilizados em estudos experimentais e, mesmo, em um número significativo de pacientes 4 é aquele originariamente descrito por Pierce-Donachy (desenvolvido pela Thoratec Laboratories Corporation), modelo de fluxo pulsátil, pneu-

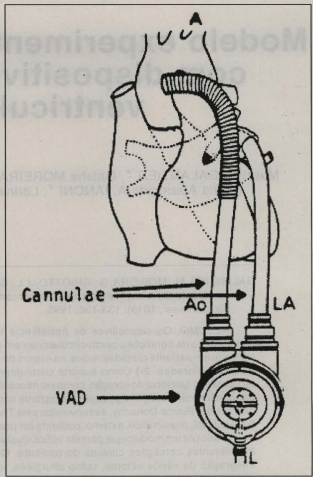

Fig. 1

mático e externo, para suporte uni ou biventricular. E constituído por um ventrículo protético, cânulas para conexão atrial, ventricular e arterial e um módulo de acionamento pneumático.

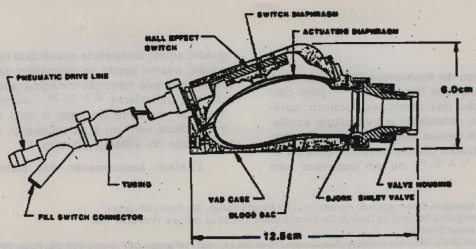

Fig. 2 
GALANTIER, M.; MOREIRA, G.; GHIOTTO, J. L.; GALANTIER, J.; IANONI, S. A. A.; MORAES, L. L.; FÉHER, J. - MOdelo experimental para treinamento com dispositivo de assistência ventricular. Rev. Bras. Cir. Cardiovasc., 10 (3): 133-138, 1995.

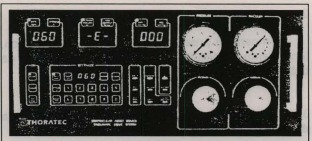

Fig. 3

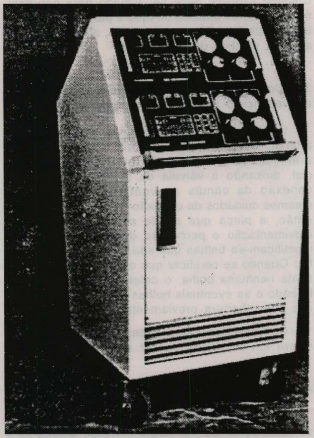

Fig. 4

O ventrículo propriamente dito (Figuras 1 e 2) é composto por um envoltório rígido de polissulfona, contendo duas câmaras. Uma sangüinea, sacular, elástica, com volume de $65 \mathrm{ml}$ e paredes de um multipolímero (BPS 215), e outra pneumática. Ambas são separadas por um diafragma de poliuretano. Sobre este diafragma há um sensor eletromagnético que é acionado toda vez que a câmara sangüínea é totalmente preenchida, determinando uma sístole mecânica imediata (quando o sistema está regulado para volume). $O$ ventrículo apresenta duas valvulas mecânicas tipo Björk-Shiley com disco pivotante de Delrin.

O módulo de acionamento pneumático (Figuras 3 e 4) permite injeção ou aspiração de ar para a camara pneumática, com conseqüente esvaziamento (sistole) e enchimento (diástole) da câmara sangüinea. Apresenta três modos de acionamento, - assíncrono (freqūência fixa ou regulável), o sincrono (praticamente em desuso, com sincronismo ao QRS ou a uma fonte externa de estímulo) e o de volume (Fill-to-Empty). Este último ajuda automaticamente a frequeência do D A V de acordo com o retorno venoso ${ }^{6}$. O módulo dispōe de duas unidades de acionamento (para assistência uni ou biventricular) e sistema de baterias permitindo autonomia de cerca de $40 \mathrm{~min}$ em caso de falta de energia, ou para deambulação do paciente.

O treinamento para utilização desse equipamento ${ }^{18}$, ou de outros modelos, requer a participaçăo não só da equipe cirúrgica mas também de médicos (intensivistas, cardiologistas) e paramédicos (enfermeiras, biomédicos, eletrotécnicos), devendo ser considerados não só os aspectos técnicos de implante, como também manuseio e controle do equipamento durante a evoluçăo do paciente. Esse treinamento em laboratórios de cirurgia experimental requer a utilização de animais de maior porte (bezerros, carneiros etc), os quais, além de custo elevado, săo de obtençăo e manuseio complicados, não permitindo o uso freqūente, com o qual haveria reciclagem adequada para os diferentes participantes.

Visando permitir esse treinamento em condiçöes mais simples, no qual aspectos técnicos de implante, retirada de ar do circuito, manuseio do módulo de acionamento, reproduçāo de situaçōes clínicas e emergenciais com o uso do equipamento, os autores desenvolveram um modelo experimental.

\section{MATERIAL E MÉTODOS}

\section{1) Descriçāo do Modelo Experimental}

Utiliza-se coração bovino, retirado de tal forma a preservar veias cavas, veias pulmonares, tronco pulmonar, aorta ascendente e croça de aorta. 0 mesmo é dissecado cuidadosamente e as veias pulmonares são suturadas com Prolene 5.0, exceto uma, através da qual é introduzida cânula $51 \mathrm{~F}$, fixada por sutura em bolsa com fio 2.0. A seguir, a aorta, após emergência do tronco braquiocefálico, é canulada com conector de $1 / 2$ polegada, fixado com várias ligaduras de cordoné. 
A linha arterial (tubo de $3 / 8$ de polegada) vai a um reservatório plástico e a cânula venosa é conectada através de tubo de $3 / 8$ de polegada a bomba de roletes de máquina de circulaçăo extracorpórea, a qual recebe tubo de retorno do reservatório plástico, fechando o circuito:

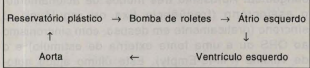

Pode-se monitorar pressăo em átrio esquerdo e aorta.

\section{2) Aspectos Técnicos de Implante do D A V}

\section{2a - Canulaçăo do ventrículo esquerdo}

Coloca-se um ponto de reparo na ponta do ventrículo esquerdo. São passados cerca de oito a dez pontos em $U$ de Ethibond 1, com agulha de 4 $\mathrm{cm}$, todos ancorados em placas de Dacron, de modo circular em redor do ponto de reparo, procurando penetrar no miocárdio a cerca de $5 \mathrm{~cm}$ do ápex e sair a $1.5-2 \mathrm{~cm}$ do mesmo. Abertura da cavidade ventricular com bisturi, exame da cavidade para verificação e eliminação de coágulos. Introduçāo de cânula especial, a qual tem um anel de Teflon para colocaçăo dos pontos ventriculares. Esses pontos, amarrados, fixam a cânula em posição adequada.

\section{2b - Anastomose do tubo à aorta}

Como a origem do tronco braquiocefálico bovino é baixa em relação à aorta, costumamos utilizar sua abertura para sutura de tubo de Dacron. Na realidade, por questǒes de hemostasia, utilizamos, neste modelo experimental, um tubo adaptado de pericárdio bovino $\left({ }^{*}\right)$.

\section{3) Retirada do Ar do Sistema - Conexão das Cânulas}

3a - Retirada de ar do circuito extracorpóreacoração bovino

É feita por recirculação, manuseio e massagem do coraçāo.

- Pericárdio bovino, Biocor.

\section{3b - Enchimento do ventrículo artificial}

O mesmo é preenchido com solução fisiológica, lentamente e variando as posições para retirada de bolhas maiores.

\section{3c - Conexão da cânula do VE (cânula de entrada do ventrículo artificial}

Esta conexão é feita simultaneamente à injeção de solução fisiológica para remoção parcial do ar contido no tubo e no conector do ventrículo artificial. Cuidado especial deve ser tomado para não danificar o material plástico do tubo e para que o mesmo encaixe perfeitamente na conexăo, antes de apertar a rosca plástica de fixação.

\section{3d - Conexão da cânula de aorta (cânula de saída do ventrículo artificial) e retirada final ao ar}

Inicialmente, faz-se pequena abertura no tubo arterial, coloca-se um ponto em $U$ de Prolene 5.0 e introduz-se um cateter $6 \mathrm{~F}$ até o ventrículo artificial, deixando a válvula de saída insuficiente. A conexāo da cânula ao ventrículo obedece aos mesmos cuidados da conexão de entrada. Solta-se, então, a pinça que ocluía o tubo arterial. Com movimentação e percussão do ventrículo artificial identificam-se bolhas que sāo aspiradas pelo cateter. Quando se certificar que o ventrículo não apresenta nenhuma bolha, o cateter é lentamente mobilizado $e$ as eventuais bolhas do tubo são eliminadas pela abertura previamente realizada.

Completadas todas essas etapas, o sistema estará em condiçőes de ser acionado.

\section{4) Manuseio do Módulo de Acionamento}

Após a conexäo do sensor eletromagnético e do tubo de ar ao ventrículo artificial, aciona-se a bomba arterial da máquina de extracorpórea com um fluxo de $3,5 \mathrm{l} / \mathrm{min}$ a $4,0 \mathrm{l} / \mathrm{min}$ e abre-se a pinça da cânula do VE. Regula-se a pressão positiva do módulo em $150 \mathrm{mmHg}$ a $180 \mathrm{mmHg}$ e a pressão de vácuo em $20 \mathrm{mmHg}$ a $25 \mathrm{mmHg}$.

\section{4a - Funcionamento em modo assíncrono}

É o sistema habitualmente utilizado no início do procedimento e também antes da interrupçăo do sistema, em casos de recuperação miocárdica adequada. Normalmente, inicia-se com uma freqũência fixa mais baixa ( 40 a 50 sistoles $/ \mathrm{min}$ ) e com uma porcentagem de sistole correspondente à metade da frequeência $(20 \%$ a $25 \%)$, com a qual teremos um 
GALANTIER, M.; MOREIRA, G.; GHIOTTO, J. L.; GALANTIER, J.; IANONI, S. A. A.; MORAES, L. L.; FEHER, J. - Modelo experimental para treinamento com dispositivo de assistencia ventricular. Rev. Bras, Cir. Cardiovasc., 10 (3): 133-138, 1995.

tempo de ejeção em torno de $300 \mathrm{mseg}$. Ligado o sistema, observa-se que a sístole e a diástole do ventriculo artificial ocorrem adequadamente. $O$ sistema é mantido nessas condiçōes por um período de $5 \mathrm{~min}$ a $10 \mathrm{~min}$, quando se aumenta, progressivamente, a frequêencia para 55 até 60 sistoles por minuto e a porcentagem de sístole até $30 \%$.

\section{4b - Funcionamento em modo volume (fill to empty)}

Uma vez atingida uma freqüência fixa de 60 sistoles/min e com funcionamento estável (pode-se elevar o fluxo da bomba para 4,0 $\mathrm{l} / \mathrm{min}$ - 4,5 $\mathrm{l} / \mathrm{min}$ ou aumentar o vácuo para $30 \mathrm{mmHg}-35 \mathrm{mmHg}$ ), procura-se verificar, no painel do módulo, se o sinal de enchimento do ventrículo artificial está presente de modo constante (sinal Fill), o mesmo acontecendo com o número 65 (volume sistólico). Isso significa que o ventrículo artificial, para essa freqüência, está se enchendo totalmente em cada sístole. Nessas condiçőes, passa-se ao modo de acionamento por volume, observando-se as alteraçōes de freqũencia ( $e$, automaticamente, da porcentagem de sístole) em diversas condições de funcionamento do sistema (aumento ou reduçăo do fluxo da bomba arterial, aumento ou diminuiçăo do vácuo, aumento ou redução da resistência periférica etc).

\section{5) Reprodução de Situações Clínicas e Emergenciais}

Com o sistema funcionando em todo volume, certas situaçōes clínicas, como hipervolemia, hipovolemia, aumento da resistência periférica, reduçăo da resistência periférica etç, ou situaçōes emergenciais (acotovelamento de entrada e saida, desconexāo do sistema Hall, desconexāo do tubo pneumático, falha na energia etc) podem ser simuladas.

\section{RESULTADOS}

A montagem do modelo experimental, os detaIhes técnicos de implante, conexöes, retirada de ar e o entendimento do manuseio do módulo de acionamento puderam ser realizados de forma reprodutivel e com baixo custo. $O$ laboratório nāo requer recursos especializados. A reproduçāo de situaçōes clinicas, principalmente no que se refere ao auto-ajuste da freqüência e débito também foi possivel, notando-se aumento da freqüência do ventriculo artificial quando do aumento do débito pela bomba arterial, com o aumento da resistência, com o aumento da pressāo negativa, ou, ainda, quando se massageava o coração e, ao, contrário, redução desta freqüência em situaçōes inversas. Verificaçāo dos diferentes alarmes (falha de enchimento, pressão baixa, sinal e etc) em situaçōes artificiais também foi nitidamente observada.

\section{COMENTÁRIOS}

O Dispositivo de Assistência Ventricular Pneumático Thoratec vem sendo utilizado clinicamente desde 1982 (Pennington - St. Louis) e, em maio de 1994, já havia mais de 400 pacientes que receberam suporte tanto como ponte para transplante (289 pacientes, sendo $180-62(3 \%)$ submetidos a transplante, dos quais sobreviveram $153(85 \%)$ como em assistência circulatória em situações agudas (118 pacientes, sendo que $44(37,3 \%)$ puderam ser desconectados do sistema - dos quais $26(22 \%)$ sobreviveram ${ }^{5}$. Inúmeros pacientes usaram o D A $\checkmark$ por mais de 1 mês. Os resultados animadores com esse e outros tipos de sistema têm levado vários pesquisadores a procurar modelos implantáveis de tal forma a manter o paciente em espera de transplante ou da recuperaçāo do seu coraçăo, até mesmo fora do ambiente hospitalar, em convivio social e familiar mais adequado $11,12,16,19$.

Em nosso meio, os trabalhos pioneiros do InCór, no desenvolvimento de um sistema pneumático externo, evoluíram para utilização clínica em alguns pacientes, sendo o primeiro deles sustentado por alguns dias, recebendo transplante a seguir e com bom resultado ${ }^{17}$. Também são relatadas tentativas isoladas com uso de bombas centrífugas, quer em suporte pós cardiotomia ou como ponte para transplante, porém os resultados não foram favoráveis. Acreditamos que a indicação para utilização clínica deva ser reestruturada, visando, principalmente, à precocidade na mesma, quando as condições do paciente sejam mais satisfatórias.

Por outro lado, as dificuldades para treinamento em animais de maior porte, em laboratórios especializados, contribuem para que não se alcance uma padronização adequada para manuseio e controle dos pacientes. O método descrito, por permitir o treinamento em condiçōes bastante simples e com acesso aos vários tipos de profissionais envolvidos no sistema, pode permitir não só essa padronização adequada, como também uma divulgação mais eficiente e uma possibilidade de reciclagem periódica de todo o sistema. 
GALANTIER, M.; MOREIRA, G.; GHIOTTO, J. L.; GALANTIER, J.; IANONI, S. A. A.; MORAES, L. L.; FÉHER, J. - MOdelo experimental para treinamento com dispositivo de assistência ventricular. Rev. Bras. Cir. Cardiovasc., 10 (3): $133-138,1995$.

\section{RBCCV $44205-269$}

GALANTIER, M.; MOREIRA, G.; GHIOTTO, J.L.; GALANTIER, J.;IANONI, S. A. A.; MORAES, L. L.; FÉHER, J. - Experimental ventricular assist device to maintain cardiocirculatory conditions ReV. Bras. Cir. Cardiovasc, 10 (3): 133-138, 1995.

ABSTRACT: The Ventricular Assist Devices (V A D) have been used to maintain the cardiocirculatory conditions in patients waiting for a heart transplantation (bridge to transplant) or in patients with an acute myocardial failure during a period enough to allow a myocardial recovery. The Pierce-Donachy System (Thoratec Laboratories Corporation) is one of those devices, a pulsatile flow model, pneumatic, external, used as uni or biventricular support. The training program for different groups of surgeons, phisicians, nurses and thecnicians is difficult in many situations, mainly when it is necessary to repeat those experiences several times to permit to those groups to be more familiar with the equipment. The authors developed an experimental model (utilizing a bovine heart and an arterial pump for extracorporeal circulation) to allow a simplified way of training, analising surgical aspects, use of the drive console and simulating clinical and emergencies situations.

DESCRIPTORS: Ventricular assistance, experimental. Assisted circulation, mechanic, experimental.

\section{REFERÊNCIAS BIBLIOGRÁFICAS}

1 COOLEY, D. A.; LIOTTA, D.; HALLMAN, G. L.; MILAM, J. D. - Orthotopic cardiac prosthesis for two-staged cardiac replacement. Am. J. Cardiol., 24: 723-730, 1969.

CURTIS, J. J. - Centrifugal mechanical assist for post cardiotomy ventricular failure. Semin. Thorac. Cardiovasc. Surg., 6: 140-146, 1994.

3 DONACHY, J. H.; LANDIS, D. L.; ROSEMBERG, G.; PIERCE, W. S. - Design and evaluation of a left ventricular assist device: the angle port pump. In: UNGER, F. (ed.) - Assisted circulation. Berlin, Springer - Verlag, 1979.

4 FARRAR, D. J. - 500 VAD patients. Thoratec's Heartbeat, 8: $1-6,1994$

FARRAR, D. J. - Thoratec VAD. Clinical Summary, May 1994. Thoratec's Heartbeat, 8: 2-6, 1994.

6 FARRAR, D. J.; COMPTON, P. G.; LANSON, J. H.; HERSHON, J. J.; HILL, J. D. - Control modes of a clinical ventricular assist device. IEEE Eng. Med. Biol, Magazine, p. 19-25, March 1986.

7 FRAZIER, O. H. - The development of an implantable, portable, eletrically powered left ventricular assist devices. Sem. Thorac. Cardiovasc. Surg., 6: 181 . $187,1994$.

HILL, J. D.; FARRAR, D. J.; HERSHOW, J. J.; COMPTON, P. G.; AVERY, G. J.; BRENT, B. N. Use of a prosthetic ventricle as a bridge to cardiac transplantation for post infarction cardiogenic schock. New Engl. J. Med., 314: 626-628, 1986.

HOLMAN, W. L.; BOURGE, R. C.; KIRKLIN, J. L. . Circulatory support for seventy days with resolution of acute heart failure. J. Thorac. Cardiovasc. Surg., 102: 932-934, 1991.

LIOTTA, D.; HALL, C. N.; WALTER, S. H.; DE BAKEY, M. E. - Prolonged assisted circulation during and after cardiac or aortic surgery. Am. J. Cardiol, 12: $399-405,1963$
11 LOISANCE, D.; DELEUZE, P. H.; MAZZUCOTELLI, J. P.; ABE, Y.; BERNERAIS, P.; DUBOIS-RANDE, J. L. - The inicial experience with the wearable Baxter Novacor ventricular assist system - J. Thorac. Cardiovasc., Surg., 108: 176-177, 1994.

12 MCCARTHY, P. M. \& SABIK, J. F. - Implantable circulatory support devices as a bridge to heart transplantation - Semin. Thorac. Cardiovasc. Surg., 6: $174-180,1994$.

13 MILLER, L. N. - Mechanical assist devices in intensive cardiac care. Am. Heart J., 121: 1887-1892, 1991.

14 PAE Jr., W. E. \& PIERCE, W. S. - Temporary ventricular assistance in acute myocardial infarction and cardiogenic shock. Chest, 79: 692-695, 1981.

15 PENNINGTON, D. G.; KANTER, K. R.; Mc BRIDE, L. R.; WILLMAN, V. - Seven years experience with the Pierce Donachy ventricular assist device. J. Thorac. Cardiovasc. Surg., 96: 901-911, 1988.

16 SAPIRSTEN, J. S.; PAE, W. E.; ROSENBERG, G.; PIERCE, W. S. - The development of permanent circulatory support systems. - Sem. Thorac. Cardiovasc. Surg., 6: 188-194, 1994.

17 STOLF, N. A. G.; LERNER, A. A.; FERNANDES, P. M. P.; JATENE, A. D. - Circulaçắo assistida mecânica como ponte para transplante. In: 25 anos de transplante cardiaco no Brasil. Săo Paulo, Departamento Científico do Centro Acadêmico Oswaldo Cruz. Faculdade de Medicina da USP, 1993 .p. 29-35.

THORATEC LABORATORIES CORPORATION: VAD Training Course. Califórnia, Thoratec, 1990.

19 VETTER, H. O.; SCHMITZ, C.; KAULBACH, H. G.; FRATER, R. W. M. - Experience with the Novacor left ventricular assist system as a bridge to cardiac transplantation, including the new wearable system. J. Thorac. Cardiovasc. Surg., 109: 74-80, 1995.

20 ZUMBRO, G. L.; SHEARER, G.; KITCHENS, W. R.; GALLOWAY, R. F. - Mechanical assistance for biventricular failure following coronary bypass operation and heart transplantation. Heart Transplant. 4: $348-352,1985$. 